\title{
Fecal Microbiota Alterations and Small Intestinal Bacterial Overgrowth in Functional Abdominal Bloating/Distention
}

\author{
Choong-Kyun Noh and Kwang Jae Lee* \\ Department of Gastroenterology, Ajou University School of Medicine, Suwon, Gyeonggi-do, Korea
}

\begin{abstract}
Background/Aims
The pathophysiology of functional abdominal bloating and distention (FABD) is unclear yet. Our aim is to compare the diversity and composition of fecal microbiota in patients with FABD and healthy individuals, and to evaluate the relationship between small intestinal bacterial overgrowth (SIBO) and dysbiosis.
\end{abstract}

\section{Methods}

The microbiota of fecal samples was analyzed from 33 subjects, including 12 healthy controls and 21 patients with FABD diagnosed by the Rome IV criteria. FABD patients underwent a hydrogen breath test. Fecal microbiota composition was determined by $16 \mathrm{~S}$ ribosomal RNA amplification and sequencing.

\section{Results}

Overall fecal microbiota composition of the FABD group differed from that of the control group. Microbial diversity was significantly lower in the FABD group than in the control group. Significantly higher proportion of Proteobacteria and significantly lower proportion of Actinobacteria were observed in FABD patients, compared with healthy controls. Compared with healthy controls, significantly higher proportion of Faecalibacterium in FABD patients and significantly higher proportion of Prevotella and Faecalibacterium in SIBO $(+)$ patients with FABD were found. Faecalibacterium prausnitzii, was significantly more abundant, but Bacteroides uniformis and Bifidobacterium adolescentis were significantly less abundant in patients with FABD, compared with healthy controls. Significantly more abundant Prevotella copri and F. prausnitzii, and significantly less abundant B. uniformis and B. adolescentis were observed in SIBO (+) patients, compared with healthy controls.

\section{Conclusion}

The fecal microbiota profiles in FABD patients are different from those in healthy controls, particularly in SIBO (+) patients, suggesting a role of gut microbiota in the pathogenesis of FABD.

(J Neurogastroenterol Motil 2020;26:539-549)

Key Words

Faecalibacterium prausnitzii; Abdominal bloating and distention; Microbiota; Prevotella copri

Received: April 17, 2020 Revised: June 1, 2020 Accepted: July 3, 2020

(a) This is an Open Access article distributed under the terms of the Creative Commons Attribution Non-Commercial License (http://creativecommons. org/licenses/by-nc/4.0) which permits unrestricted non-commercial use, distribution, and reproduction in any medium, provided the original work is properly cited.

*Correspondence: Kwang Jae Lee, MD, PhD Department of Gastroenterology, Ajou University School of Medicine, 164, World Cup-ro, Yeongtong-gu, Suwon, Gyeonggi-do 16499 , Korea

Tel: +82-31-219-5119, Fax: +82-31-219-5999, E-mail: kjl@ajou.ac.kr 


\section{Introduction}

Abdominal bloating is a common symptom defined as a subjective feeling of abdominal pressure regardless of the presence of objective abdominal distention. ${ }^{1}$ Abdominal bloating is commonly accompanied in patients with functional gastrointestinal disorders such as irritable bowel syndrome (IBS), ${ }^{1-3}$ functional dyspepsia, ${ }^{4}$ and functional constipation. ${ }^{5}$ According to the Rome IV criteria, functional abdominal bloating and distention (FABD) can be diagnosed when there are insufficient criteria for a diagnosis of IBS, functional constipation, functional diarrhea, or postprandial distress syndrome. ${ }^{6}$

The pathophysiologic mechanisms of FABD are not fully understood, but believed to be multifactorial. The plausible mechanisms include increased luminal contents or gas in the intestine, small intestinal bacterial overgrowth (SIBO), imbalance of gut microbiota, food intolerance, visceral hypersensitivity, and decreased abdominal capacity induced by abnormal viscerosomatic responses or anatomical changes. ${ }^{6-8}$ Among these mechanisms, increased endogenous gas levels may be associated with dysbiosis and/or SIBO.${ }^{9,10}$ However, data regarding SIBO and dysbiosis in patients with FABD are lacking and their association has not been fully investigated. The cause-and-effect relationship between gut microbiota alterations, such as SIBO and fecal dysbiosis, and FABD has not been clearly determined. However, studies have shown that the administration of rifaximin to patients with IBS without constipation improved IBS symptoms and bloating. ${ }^{11}$ Similarly, administration of probiotics is reported to reduce the symptom severity of patients with functional abdominal bloating. ${ }^{12}$ Although studies suggest an association of gut microbiota alterations with the pathogenesis of FABD, specific gut microorganisms associated with FABD have not yet been reported.

Therefore, this prospective study was conducted to compare the diversity and composition of fecal microbiota in patients with FABD and healthy individuals, and to evaluate the relationship between SIBO and dysbiosis.

\section{Materials and Methods}

\section{Study Population and Design}

This prospective, single-center study was conducted at Ajou University Hospital (Suwon, Gyeonggi-do, Korea). The study protocol was approved by the institutional review board (Approval No.
AJIRB-BMR-SMP-17-472). All participants provided written informed consent.

Patients meeting the Rome IV criteria for FABD were enrolled in the study. The criteria included: (1) recurrent feelings of bloating or visible distention for at least 3 days per month in the last 3 months, (2) onset of symptoms at least 6 months prior to presentation, and (3) insufficient criteria to establish diagnosis of IBS or functional dyspepsia. ${ }^{6}$ Every subject completed a gastrointestinal symptom questionnaire. Degrees of abdominal distention and bloating sensation were measured by a visual analog scale score. General demographic information, past medical and operation history, medications (antibiotics, probiotics, or other medications), and Bristol stool form scales were recorded. ${ }^{13}$ All subjects underwent the hydrogen breath test (HBT) and were subsequently classified into $\mathrm{SIBO}(-)$ and $\mathrm{SIBO}(+)$ groups.

Twelve healthy volunteers without organic or systemic diseases or chronically recurrent abdominal symptoms participated in the study as healthy controls. Exclusion criteria included past history of medical diseases or operation, use of medications affecting gut microbiota in the last 6 months including antibiotics and probiotics, and recurrent abdominal symptoms in the last 3 months. The ethnicity of all study participants including patients with FABD and healthy volunteers was the Korean race.

\section{Hydrogen Breath Tests}

HBT was performed after an overnight fast. Subjects were asked to abstain from consuming fiber and slowly absorbed carbohydrates (such as bread and potato) the night before the test, as these delay hydrogen excretion in the breath. ${ }^{14,15}$ Cigarette smoking and exercise were avoided 2 hours before and during the test. Discontinuation of pro-motility and anti-motility medications, antibiotics, and other drugs that can affect gut microbiota for at least 2 weeks prior to the HBT was mandated. Subjects brushed their teeth and rinsed their mouths with antiseptic mouth wash and tap water prior to the test to eliminate an early hydrogen peak due to oral bacterial action on the test sugar. ${ }^{14,15}$ End-expiratory breath samples were collected in bags. Fasting breath hydrogen concentration was measured 3 to 4 times at the start of the test. The average value was recorded as the basal breath hydrogen level. Subsequently, each subject ingested $10 \mathrm{~g}$ of lactulose, and HBT was performed every 15 minutes for 2 to 4 hours. Positive results required an elevated breath hydrogen concentration within 90 minutes, which showed 2 distinct peaks and/or an increase exceeding $20 \mathrm{ppm}^{15}$ 


\section{Fecal Sample Collection, DNA Extraction, and Sequencing}

Fecal samples collected in a centrifuge tube were stored at $-20^{\circ} \mathrm{C}$ and then transferred to $-80^{\circ} \mathrm{C}$ freezer within 24 hours for analysis. DNA was extracted from the fecal samples within 48 hours after collection using a DNeasy PowerSoil Kit (Qiagen, Hilden, Germany) according to the manufacturer's instructions. Extracted DNA was quantified using a Quant-IT PicoGreen assay (Invitrogen, Carlsbad, CA, USA) according to the manufacturer's instructions. The sequencing library was prepared according to the 16S Metagenomic Sequencing Library protocol (Illumina Inc, San Diego, CA, USA) to amplify the V3 and V4 regions. The methods for library purification, verification, and sequencing have been included in the supplementary materials. Quality filtering and operational taxonomic unit selection are also described in the supplementary materials.

\section{Diversity Analysis and Taxonomic Comparisons}

Alpha diversity was calculated using Quantitative Insights Into Microbial Ecology (QIIME) to determine species diversity, evenness, and richness of the microbiome in each sample. Chao1, Shannon, and inversed Simpson index were obtained for each sample, and rarefaction curves were generated to examine species diversity information among samples and groups. For beta diversity analysis, relationships between samples were analyzed using principal coordinate analysis and unweighted pair group method with arithmetic mean tree. The results were visualized using FigTree (version 1.4.4) (http://tree.bio.ed.ac.uk/software/figtree/). ${ }^{16}$ Dissimilarity between samples was calculated based on weighted UniFrac distances. Taxo-
Table. Baseline Characteristics of Study Subjects

\begin{tabular}{|c|c|c|c|}
\hline Variables & $\begin{array}{c}\mathrm{HC} \\
(\mathrm{n}=12)\end{array}$ & $\begin{array}{l}\text { FABD } \\
(n=21)\end{array}$ & $P$-value \\
\hline Gender (n [\%]) & & & 0.839 \\
\hline Male & $8(66.7)$ & $13(61.9)$ & \\
\hline Female & $4(33.3)$ & $8(38.1)$ & \\
\hline Age (yr) & & & 0.012 \\
\hline Mean $\pm \mathrm{SD}$ & $34.5 \pm 3.8$ & $62.1 \pm 14.7$ & \\
\hline Range & $33-45$ & $22-81$ & \\
\hline $\operatorname{BMI}\left(\mathrm{kg} / \mathrm{m}^{2}\right)$ & & & 0.910 \\
\hline Mean $\pm \mathrm{SD}$ & $23.0 \pm 2.9$ & $24.2 \pm 2.7$ & \\
\hline Medical history (n [\%]) & & & 0.122 \\
\hline Hypertension & $0(0.0)$ & $5(23.8)$ & \\
\hline Diabetes & $0(0.0)$ & $5(23.8)$ & \\
\hline Old CVA & $0(0.0)$ & $0(0.0)$ & \\
\hline Smoking history $(\mathrm{n}[\%])$ & & & 0.839 \\
\hline None & $9(75.0)$ & $16(76.2)$ & \\
\hline Past & $0(0.0)$ & $3(14.3)$ & \\
\hline Current & $3(25.0)$ & $2(9.5)$ & \\
\hline \multicolumn{4}{|l|}{ Alcohol history (n [\%]) } \\
\hline None & $2(16.7)$ & $14(66.7)$ & 0.671 \\
\hline Social & $10(83.3)$ & $6(28.6)$ & \\
\hline More than twice a week & $0(0.0)$ & $1(4.7)$ & \\
\hline Cholecystectomy (n [\%]) & $0(0.0)$ & $0(0.0)$ & 1.000 \\
\hline Lack of exercise (n [\%]) & $3(25.0)$ & $5(23.8)$ & 0.956 \\
\hline $\begin{array}{l}\text { Bloating/distention } \\
\text { (VAS score) }\end{array}$ & & & 0.001 \\
\hline Mean $\pm \mathrm{SD}$ & $1.2 \pm 0.4$ & $6.5 \pm 2.5$ & \\
\hline \multicolumn{4}{|c|}{ Hydrogen breath test (n [\%]) } \\
\hline Negative & & $12(57.1)$ & \\
\hline Positive & & $9(42.9)$ & \\
\hline
\end{tabular}

$\mathrm{HC}$, healthy control; FABD, functional abdominal bloating and distention; BMI, body mass index; CVA, cerebrovascular accident; VAS, visual analogue scale.
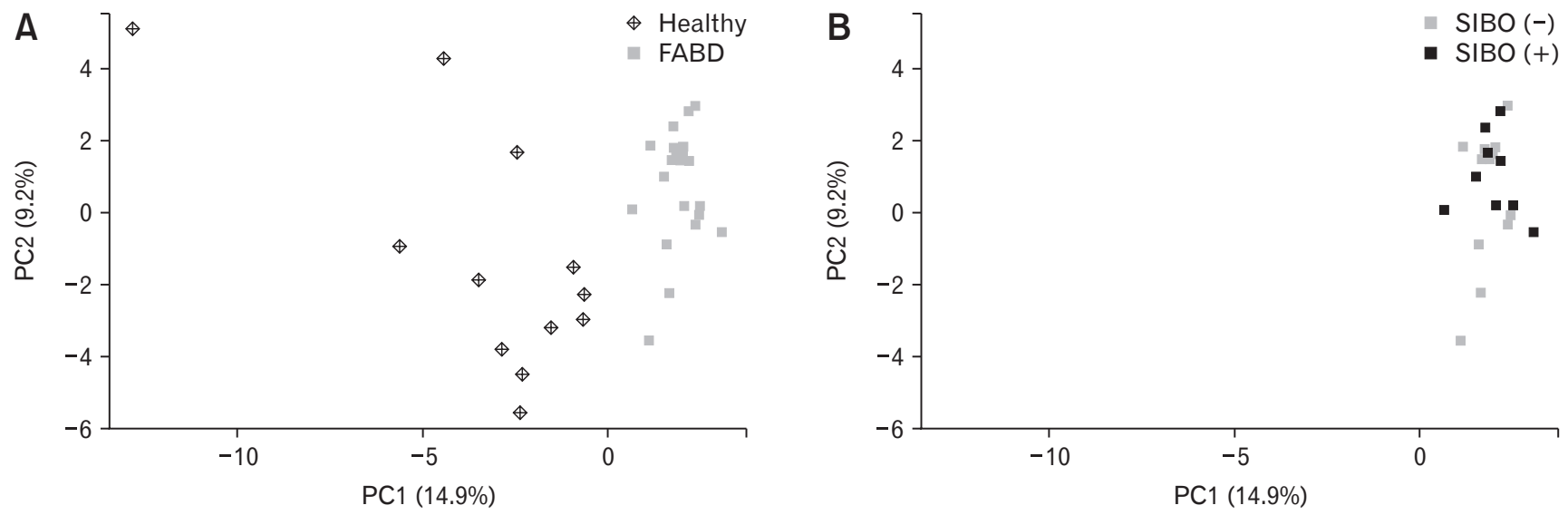

Figure 1. Unweighted UniFrac principal component analysis. (A) healthy controls vs patients with functional abdominal bloating and distention (FABD), (B) SIBO (-) patients vs SIBO (+) patients. PC, principal component; SIBO, small intestinal bacterial overgrowth. 
nomic relative abundance was determined by converting operational taxonomic unit taxonomic information using QIIME to relative abundance percentage at the phylum, genus, and species levels.

\section{Statistical Methods}

Principal component analysis (PCA) was conducted for genus and species with a relative abundance ratio (mean value of the group) $\geq 1 \%$, and for phylum at each level. For PCA, the prcomp function of the $\mathrm{R}$ package (version 3.1.2) was used to display the relationship between samples on a coordinate plane. ${ }^{17-19}$ Krona graphs (multi-layered pie charts allowing efficient visualization of taxonom- ic hierarchy) were used to visualize the relative abundance of each phylum, genus, and species using the Krona Excel Template (version 2.2). ${ }^{20}$ The Wilcoxon rank-sum test from $\mathrm{R}$ package was used for statistical analysis. ${ }^{21,22}$ To determine biomarkers differentiating healthy controls and FABD patients, linear discriminant analysis coupled with effect size measurements (LEfSe) was conducted. ${ }^{23}$ From the relative abundance ratio information, major differences between healthy controls and FABD patients were selected using the Kruskal-Wallis test. These differences were estimated through linear discriminant analysis (LDA) scores (log scale). A bar plot (LDA score) and cladogram were generated for items with a

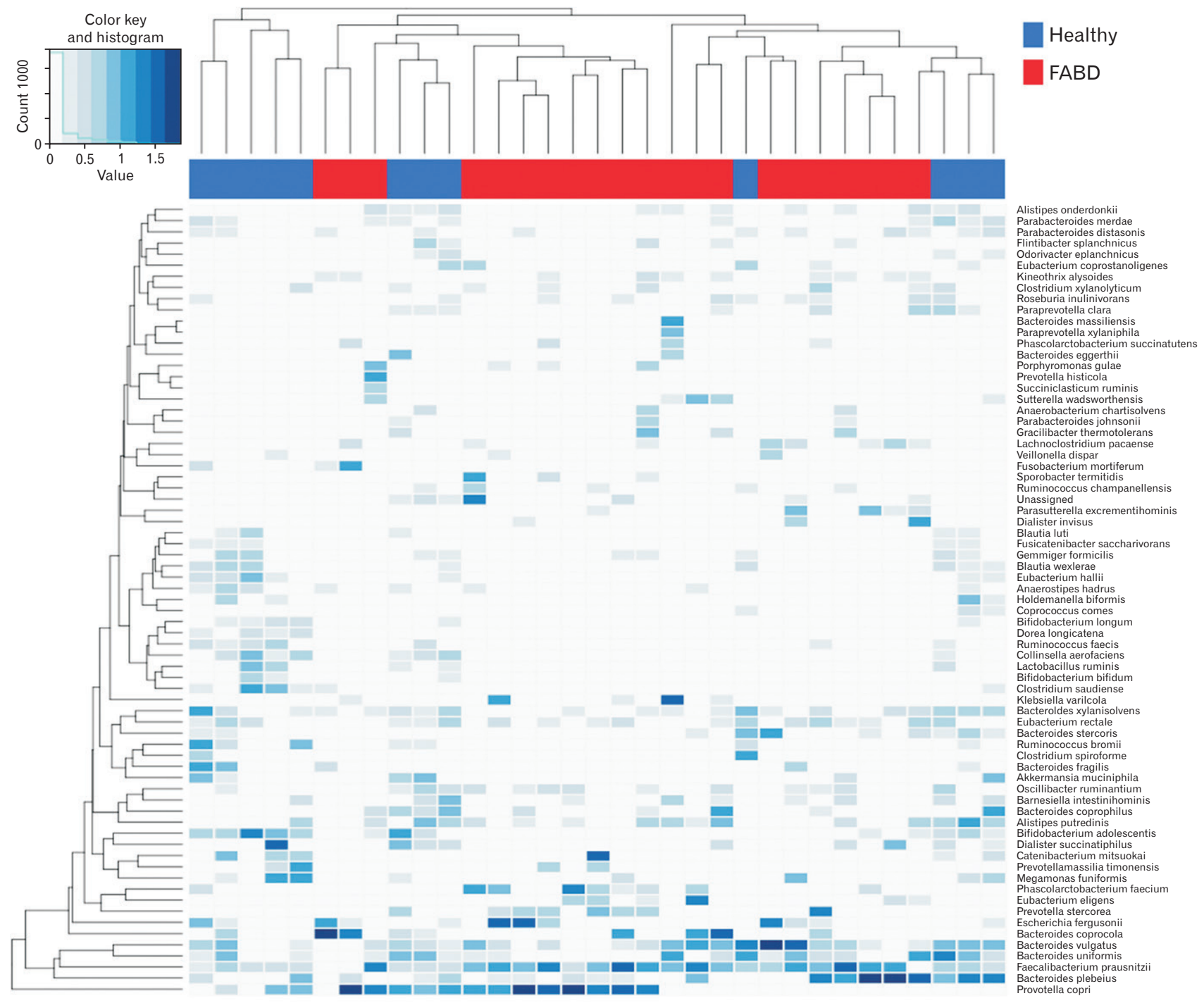

Figure 2. Heat map of taxonomic assignment of fecal samples. The colored columns in the upper part of the heat map indicate patients with functional abdominal bloating and distention (FABD) and healthy controls, and those in the lower part of the heat map indicate each subject. Taxonomic abundance is proportional to color intensity (color scale in the upper-left panel of the figure). 
$P$-value $<0.05$ (Kruskal-Wallis) and LDA score $(\log 10) \geq 2$. Statistical significance $(P<0.05)$ was evaluated for taxonomic relative abundance ratio (phylum, genus, and species) and diversity index (Shannon and inversed Simpson index).

\section{Results}

\section{Baseline Characteristics of Study Subjects}

A total of 21 FABD patients and 12 healthy controls were finally enrolled in the present study. The mean age of FABD patients was significantly higher than that of healthy controls (62.1 \pm 14.7 years vs $34.5 \pm 3.8$ years, $P=0.012$ ). Patients with FABD exhibited significantly higher scores of abdominal bloating or distention sensation $(6.5 \pm 2.5$ vs $1.2 \pm 0.4, P=0.001)$ than healthy controls. Nine patients with FABD (42.9\%) were SIBO (+). The baseline characteristics of the study population are shown in Table.

\section{Overall Fecal Microbiota Composition and Diversity}

Overall fecal microbiota composition of the FABD group differed from that of the control group, based on the PCA results (principal component $1=14.9 \%$; Fig. $1 \mathrm{~A})$. Overall fecal microbiota composition between the $\mathrm{SIBO}(+)$ and $(-)$ groups in patients with FABD did not differ significantly (Fig. 1B). Principal coordinate analysis and 3-dimensional image reconstruction (Analysis of Similarities $\mathrm{R}=0.204, P=0.020$, Supplementary Fig. 1) also illustrated differences between patients with FABD and healthy controls. Hierarchical clustering heatmaps of Pearson's correlation coefficients also revealed that the fecal microbiota composition of FABD patients differed from that of the healthy controls (Fig. 2).
Alpha diversity, measured by the Shannon $(3.350 \pm 0.181$ vs 4.764 $\pm 0.166, P<0.001)$ and inversed Simpson $(0.780 \pm 0.025$ vs $0.932 \pm 0.008, P<0.001)$ index, was significantly lower in patients with FABD than in healthy controls (Fig. 3).

\section{Relative Abundance of Bacteria Between Functional Abdominal Bloating and Distention Patients and Healthy Controls}

Relative abundance of bacteria at the phylum level differed between healthy controls and patients with FABD, as shown in the Krona chart (Fig. 4). In healthy controls, Firmicutes (44.0\%) accounted for the highest proportion, followed by Bacteroidetes (41.2\%), Actinobacteria (9.3\%), Proteobacteria (2.3\%), and Verrucomicrobia $(2.4 \%)$. The order in FABD patients was Bacteroidetes $(52.8 \%)$, Firmicutes $(35.1 \%)$, Proteobacteria (9.8\%), Fusobacteria $(0.7 \%)$, and Actinobacteria $(0.3 \%)$. The proportion of Proteobacteria in FABD patients was significantly higher than that in the healthy controls (9.8\% vs $2.3 \%, P=0.007)$. The proportion of Actinobacteria in FABD patients was significantly lower than that in healthy controls $(0.3 \%$ vs $9.3 \%, P<0.001)$.

The relative abundance of microbiota at the genus level revealed that Prevotella, Faecalibacterium, and Bacteroides were the top 3 bacterial genera in FABD patients. Prevotella (18.9\% vs $4.5 \%$, $P=0.271)$ and Bacteroides (27.8\% vs $26.3 \%, P=0.782)$ were more abundant in FABD patients than in healthy controls, but the difference was not statistically significant. In contrast, the proportion of Faecalibacterium was significantly higher in FABD patients than that in healthy controls $(12.9 \%$ vs $3.1 \%, P=0.002)$. The relative abundance of microbiota at the genus level between SIBO (-) patients and healthy controls did not differ significantly. However, the
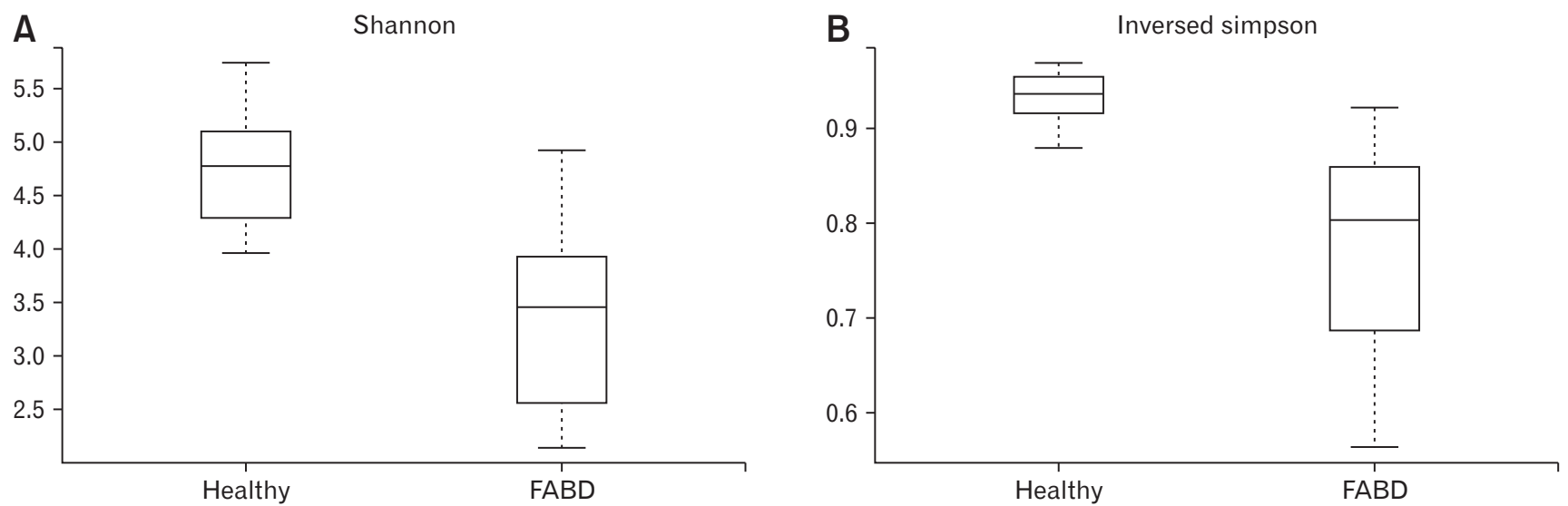

Figure 3. Comparison of overall diversity between patients with functional abdominal bloating and distention (FABD) and healthy controls. (A) Shannon index. (B) Inversed Simpson index. Statistical significance $(P<0.05)$. FABD, functional abdominal bloating and distention. 

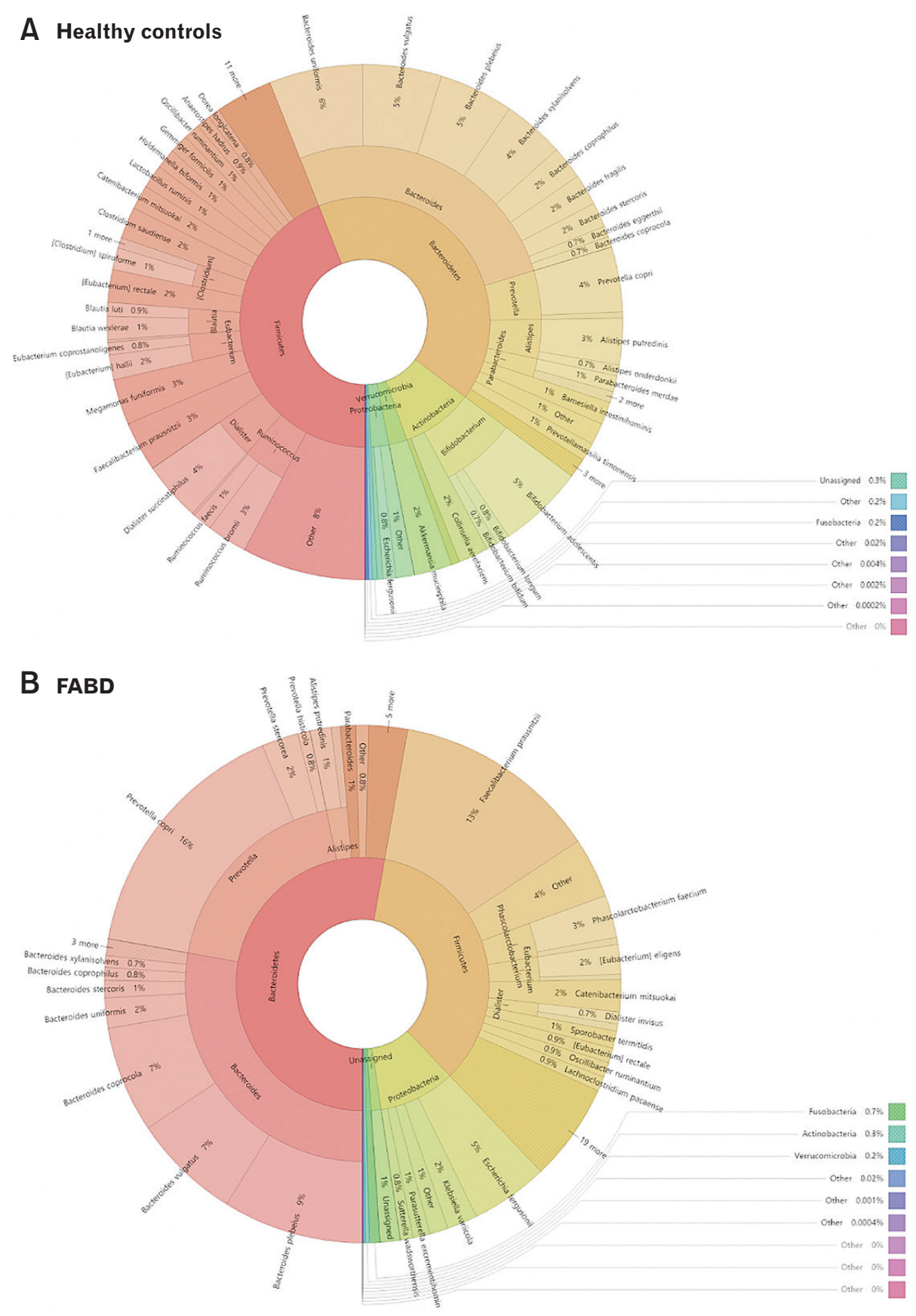

Figure 4. Krona chart illustrating differential abundance of bacteria at the phylum level. Healthy controls (A) and patients with functional abdominal bloating and distention (FABD) (B).

proportions of Prevotella (21.2\% vs $4.5 \%, P=0.024)$ and Faecalibacterium ( $16.1 \%$ vs $3.1 \%, P=0.002)$ were significantly higher in SIBO $(+)$ patients, compared with healthy controls (Fig. 5).

At the species level, Faecalibacterium prausnitzii, Bacteroides uniformis, and Bifidobacterium adolescentis differed in relative abundance between FABD patients and healthy controls (Supplementary Fig. 2). Prevotella copri demonstrated the highest relative abundance in FABD patients, which was not significantly different from its abundance in healthy controls $(15.8 \%$ vs $4.1 \%, P=0.404)$. F. prausnitzii, which was the second highest in abundance in FABD patients, was significantly more abundant in patients with FABD, compared with healthy controls (12.9\% vs $3.1 \%, P=0.002)$. On the contrary, proportions of $B$. uniformis ( $1.9 \%$ vs $5.8 \%, P=0.048$ ) and $B$. adolescentis $(0.2 \%$, vs $5.4 \%$ vs $P<0.001)$ were signifi- 


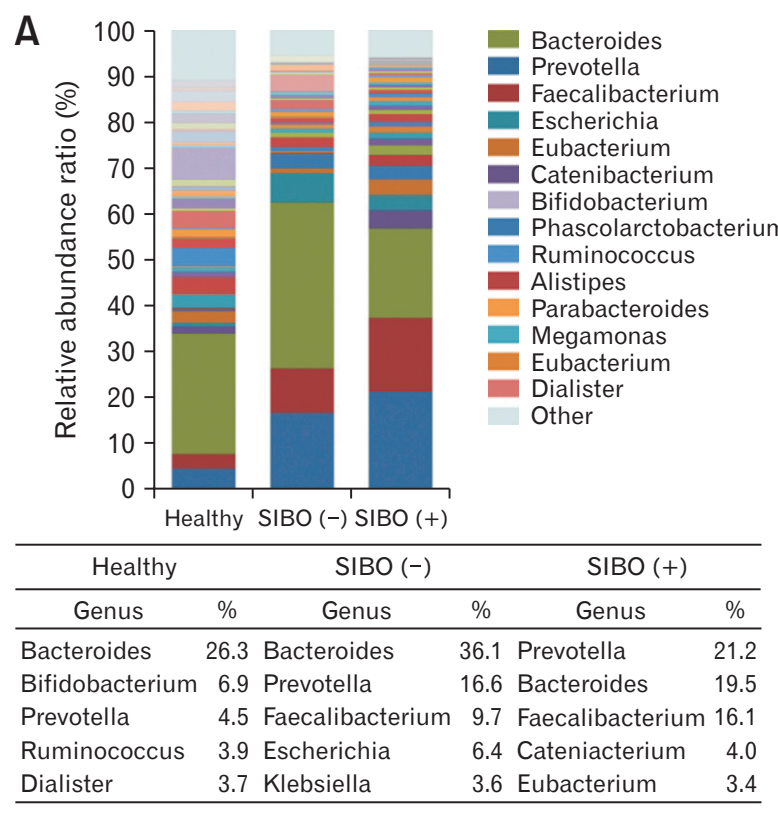

B

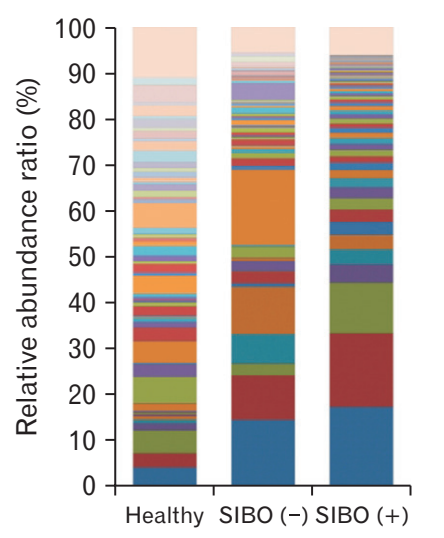

\begin{tabular}{|c|c|c|c|c|c|}
\hline \multicolumn{2}{|l|}{ Healthy } & \multicolumn{2}{|l|}{$\operatorname{SIBO}(-)$} & \multicolumn{2}{|l|}{$\operatorname{SIBO}(+)$} \\
\hline Species & $\%$ & Species & $\%$ & Species & $\%$ \\
\hline Bacteroides uniformis & 5.8 & Bacteroides plebeius & 16.5 & Prevotella copri & 17.3 \\
\hline $\begin{array}{l}\text { Bifidobacterium } \\
\text { adolescentis }\end{array}$ & 5.4 & $\begin{array}{l}\text { Prevotella copri } \\
\text { Bacteroides coprocola }\end{array}$ & $\begin{array}{l}14.4 \\
10.3\end{array}$ & $\begin{array}{l}\text { Faecallibacterium } \\
\text { prausnitzii }\end{array}$ & 16.1 \\
\hline Bacteroides vulgatus & 5.0 & Faecalibacterium & 9.7 & Bacteroides vulgatus & 11.0 \\
\hline Bacteroides plebeius & 4.7 & prausnitzii & & Catenibacterium mitsuok & ai 6.0 \\
\hline Prevotella copri & 4.1 & Escherichia fergusonii & 6.4 & Escherichia fergusonii & 4.0 \\
\hline
\end{tabular}

Figure 5. Relative abundance of bacteria at the genus (A) and species (B) levels. The top 15 bacterial genera or species that were detected are indicated. SIBO, small intestinal bacterial overgrowth.

cantly lower in patients with FABD than those in healthy controls. Among the 4 bacteria, only $B$. adolescentis $(0.2 \%$ vs $5.4 \%, P<$ $0.001)$ was significantly less abundant in SIBO $(-)$ patients than in healthy controls. Compared with healthy controls, $P$. copri $(17.3 \%$ vs $4.1 \%, P=0.031)$ and $F$. prausnitzii $(16.1 \%$ vs $3.1 \%, P=0.002)$ were significantly more abundant in $\mathrm{SIBO}(+)$ patients, while $B$. uniformis (1.4\% vs $5.9 \%, P=0.049)$ and $B$. adolescentis ( $0.2 \%$ vs $5.4 \%, P<0.001)$ were significantly less abundant in $\operatorname{SIBO}(+)$ patients.

\section{Identification of Specific Microbial Taxa Using LEfSe}

F. prausnitzii, Catenibacterium mitsuokai, Lachnoclostridium pacaense, Kineothrix alysoides, Bacteroides finegoldii, Lachnospira pectinoschiza, Acidovorax radicis, Bacteroides clarus, and Clostridium clostridioforme were more abundant in FABD patients compared with healthy controls. F. prausnitzii was the most abundant species in FABD patients compared with healthy controls (Fig. 6).

\section{Discussion}

Our results of the present study demonstrated that overall fecal microbiota composition in the FABD group differed from that in the control group. Fecal microbiota diversity was significantly lower in FABD patients than that in healthy controls. Moreover, relative abundance of bacteria significantly differed between FABD patients and healthy controls. At the phylum level, significantly higher proportion of Proteobacteria and significantly lower proportion of Actinobacteria were observed in FABD patients, compared with healthy controls. At the genus level, significantly higher proportion of Prevotella and Faecalibacterium was observed in the patient group. At the species level, the patient group, particularly the SIBO $(+)$ group, showed significantly more abundant $P$. copri and $F$. prausnitzii, and significantly less abundant $B$. uniformis and $B$. adolescentis, compared with healthy controls.

Abdominal bloating is one of the most common symptoms in functional gastrointestinal disorders. FABD is characterized by subjective symptoms of recurrent abdominal fullness, pressure, or a sensation of gas with or without objective increase in abdominal girth. ${ }^{24}$ Patients enrolled in the present study did not meet the criteria for other functional bowel disorders such as IBS. However, they may also report symptoms of mild abdominal pain and/or minor bowel movement abnormalities. Therefore, the pathophysiology of FABD is considered to be multifactorial. All patients underwent blood tests, endoscopic examinations, abdominal ultrasonography, or computed tomography to exclude organic causes for abdominal bloating or distension. Plausible causes for FABD include visceral hypersensitivity, abnormal intestinal gas transit, impaired evacuation of rectal gas, colonic fermentation, SIBO, and gut microbiota 
A

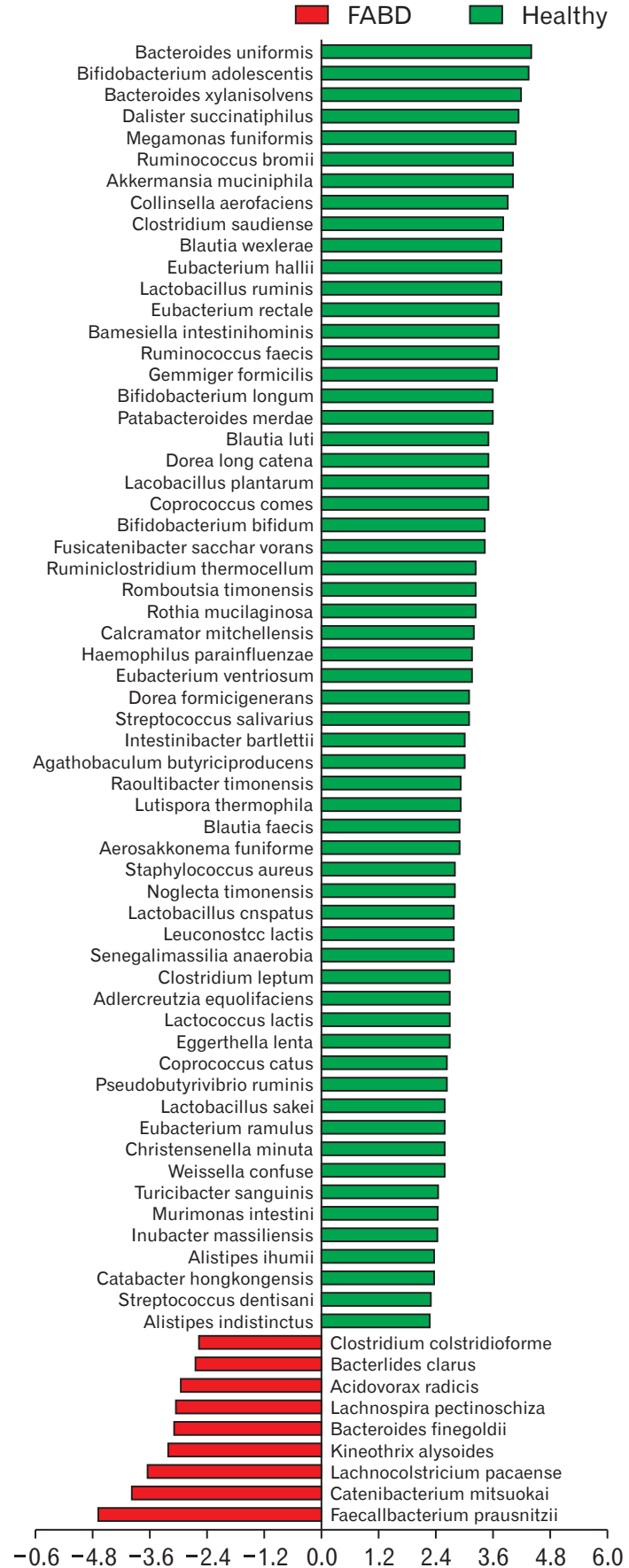

B

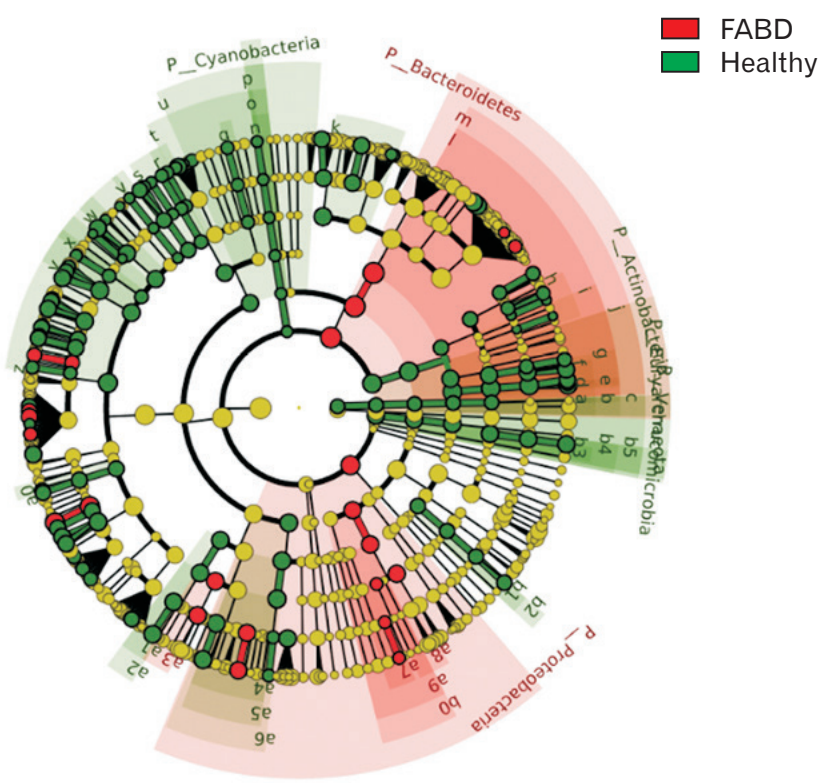

a: Family - Methanobacteriaceae

b: Order - Methanobacteriales

c: Class - Methanobacteria

d: Family - Bifidobacteriaceae

e: Order - Bifidobacteriales

f: Family - Coriobacteriaceae

g: Order - Coriobacteriales

h: Family - Unclassified

i: Order-Unclassified

j: Class - Unclassified

k: Family - Unclassified

I: Order - Bacteroidales

$\mathrm{m}$ : Class - Bacteroidia

n: Family - Unclassified

o: Order - Oscillatoriales

p: Class - Unclassified

q: Family - Staphylococcaceae

r: Family - Leuconostocaceae

s: Family - Streptococcaceae

t: Order - Lactobacillales

u: Class - Bacilli

v: Family - Catabacteriaceae

w: Family - Clostridiaceae

x: Family - Clostridiales family XIII, Incertae sedis

y: Family - Eubacteriaceae

z: Family - Lachnospiraceae

a0: Family - Peptostreptococcaceae

a1: Family - Selenomonadaceae

a2: Order - Selenmonadales

a3: Family - Acidaminococcaceae

a4: Family - Erysipelotrichaceae

a5: Order - Erysipelotrichales

a6: Class - Unclassified

a7: Family - Comamonadaceae

Figure 6. Linear discriminant analysis (LDA) coupled with effect size measurements illustrating the most differentially abundant taxa among groups at the species level (A) and Cladogram highlighting the distribution of fecal microbiota with differential abundance among groups (B). FABD, functional abdominal bloating and distention.

alterations. $^{24}$ The relevant etiopathophysiological mechanisms for FABD appear to vary from patient to patient.

It has been proposed that increased gas production or decreased gas consumption in patients with FABD may result from gut microbiota alterations. Gut microbiota alterations may result in excess gas, abnormal regulation of intestinal gas, and/or altered visceral sensation, which requires further investigation. Abnormal intraluminal fermentation by gut bacteria could lead to excessive gas 
production and bloating symptoms at least in some patients. ${ }^{25}$ The association of gut microbiota with abdominal bloating is also supported by the beneficial effect of therapeutic interventions targeting the intestinal microbiota, including antibiotics, probiotics, prebiotics, and diet low in fermentable carbohydrates. A recent study investigated the relationship between intestinal microbiota, abdominal bloating, and altered bowel patterns in a cohort of patients with IBS and healthy controls, demonstrating quantitative differences of species/operational taxonomic units between subgroups of IBS patients with and without bloating. ${ }^{26} \mathrm{~A}$ significant association between gut microbiota and bloating symptoms in IBS patients suggests a role of intestinal microbiota in the pathogenesis of bloating in IBS. The fecal microbiota alterations at the phylum, genus, and species levels in patients with FABD observed in the present study also provide evidence suggesting a role of intestinal microbiota in the pathogenesis of FABD.

In the present study, $\mathrm{SIBO}$ was diagnosed in $42.8 \%$ of patients with FABD. At the genus level, higher proportion of Prevotella and Faecalibacterium are likely to be involved in FABD, particularly in SIBO $(+)$ patients. Moreover, significantly more abundant $P$. copri and $F$. prausnitzii, and significantly less abundant $B$. uniformis and $B$. adolescentis were observed in FABD patients, particularly in SIBO $(+)$ patients, compared with healthy controls. However, the relative abundance of microbiota at the genus level between SIBO $(-)$ patients and healthy controls did not differ significantly. In contrast to $\mathrm{SIBO}(+)$ patients, only $B$. adolescentis was significantly less abundant in $\mathrm{SIBO}(-)$ patients than in healthy controls. These findings suggest that SIBO can be associated with fecal microbiota alterations. The proportion of $P$. copri and $F$. prausnitzii species was $33.4 \%$ in SIBO $(+)$ patients, which was 4.6-fold higher than that in healthy controls (7.2\%). Prevotella species are anaerobic gramnegative bacteria in phylum Bacteroidetes. ${ }^{27}$ Prevotella-enriched gut dysbiosis was reported to be associated with chronic inflammatory diseases and induced inflammation mediated by $\mathrm{T}$ helper 17 cellrelated immune responses. ${ }^{28}$ Some Prevotella strains have been suggested to be clinically important pathobionts that can promote chronic inflammation. In contrast, F. prausnitzii, an anaerobic gram-positive bacterium, is an anti-inflammatory commensal bacterium. ${ }^{29} \mathrm{~A}$ low $F$. prausnitzii count is found in colitis patients, ${ }^{30}$ and anti-inflammatory metabolites secreted by this bacterium are known to be detected in patients with Crohn's disease. ${ }^{29,31}$ The cause of paradoxical results for the abundance of $P$. copri and $F$. prausnitzii, which play opposite roles in the gut microbiome, in patients with FABD remains to be unexplored. Rather, less abundant $B$. uniformis and $B$. adolescentis may play a crucial role in the pathophysiol- ogy of FABD. A previous study showed evidence of the potential modulatory effects of $B$. uniformis on the metabolic and immune function in mice. ${ }^{32} \mathrm{~B}$. adolescentis is also known to have an immune modulatory effect. ${ }^{33}$ Communication between the gut microbiota and the host occurs through various biomolecules, nutrient signaling-independent pathways, and epigenetic mechanisms. Cross talk between altered gut microbiota derived metabolites and important cells of the gastrointestinal tract (ie, enterochromaffin cells, interstitial cells of Cajal, and resident macrophages) may be involved in the change of the gut function. It may explain the impact of gut dysbiosis on some important pathophysiological mechanisms of FABD. The changes in the microbial composition in the FABD group were significant. In the present study, the FABD group was divided into the SIBO $(+)$ and SIBO $(-)$ subgroups. The SIBO $(+)$ subgroup showed remarkable changes in the microbial composition, whereas the SIBO (-) subgroup did not. The cause-andeffect relationship between changes in relative abundance of gut bacteria and FABD requires further investigation. Moreover, the relationship between altered intestinal microbiota, SIBO or bloating symptoms warrants further investigation.

This prospective study has several limitations. While an increased relative abundance of specific bacteria in FABD patients was evident, laboratory evaluation to examine the mechanism related to fecal microbiota alterations was not performed. Second, the effect of age could not be excluded. Age can affect microbiota composition. ${ }^{34}$ However, it is very difficult to obtain healthy samples from the elderly aged population. The elderly aged persons have difficulty in strictly controlling diet, medication, and other routine life activities, that may affect gut microbiota. In the present study, diet, medication, and routine life activities of healthy controls were controlled for obtaining healthy samples. Although aging may be a factor for gut microbiota shaping, within-individual variation of gut microbiota is known to be relatively stable over time. Studies indicate that age-related gut dysbiosis may contribute to unhealthy aging and reduced longevity, indicating that samples from the aged people may not be real healthy controls. In the present study, we aimed to know the difference in fecal microbiota between patients with FABD and real healthy controls. Actually, the findings of fecal microbiota composition observed in patients with FABD are different from those related to aging. Moreover, the mean age of patients with FABD is 62 , which is relatively below that of the old age group. Third, diet is a major factor affecting gut microbiota as well as a crucial factor in the pathogenesis of abdominal bloating. ${ }^{35,36}$ Dietary factors may contribute to gut microbiota alterations in patients with FABD. Since habitual diet can be associated with bloat- 
ing symptoms, they were asked to maintain usual dietary patterns in the present study. Thus, diet was not strictly controlled. Fourth, SIBO is defined by the increased number of microbiota in the small intestine. The compositional change in microbiota related to SIBO remains yet unknown. Fecal microbiota may be affected by the small intestine as well as the large intestine. In order to confirm dysbiosis in the small intestine, the intestinal aspirates may be more appropriate rather than feces.

In conclusion, overall fecal microbiota composition in the FABD group differed from that in the control group. FABDassociated alterations of gut microbiota included lower diversity, increased abundance of $P$. copri and F. prausnitzii, and reduced abundance of $B$. uniformis and $B$. adolescentis, which were more prominent in $\mathrm{SIBO}(+)$ patients than in SIBO $(-)$ patients. Further studies to establish the role of this dysbiosis in the pathophysiology of FABD are warranted.

\section{Supplementary Materials}

Note: To access the supplementary figures mentioned in this article, visit the online version of Journal of Neurogastroenterology and Motility at http://www.jnmjournal.org/, and at https://doi. org/10.5056/jnm20080.

\section{Financial support: None.}

\section{Conflicts of interest: None.}

Author contributions: Choong-Kyun Noh: planning the study design, reviewing the data, analyzing the data, and drafting the manuscript; and Kwang Jae Lee: planning the study design, collecting the data, reviewing the data, analyzing the data, interpreting the data, drafting the manuscript, and critically revising the manuscript.

\section{References}

1. Houghton LA, Lea R, Agrawal A, Reilly B, Whorwll PJ. Relationship of abdominal bloating to distention in irritable bowel syndrome and effect of bowel habit. Gastroenterology 2006;131:1003-1010.

2. Neri M, Laterza F, Howell S, et al. Symptoms discriminate irritable bowel syndrome from organic gastrointestinal diseases and food allergy. Eur J Gastroenterol Hepatol 2000;12:981-988.

3. Kanazawa M, Miwa H, Nakagawa A, Kosako M, Akiho H, Fukudo S. Abdominal bloating is the most bothersome symptom in irritable bowel syndrome with constipation (IBS-C): a large population-based internet survey in Japan. Biopsychosoc Med 2016;10:19.
4. Kwan AC, Bao T, Chakkaphak S, et al. Validation of rome II criteria for functional gastrointestinal disorders by factor analysis of symptoms in Asian patient sample. J Gastroenterol Hepatol 2003;18:796-802.

5. Talley NJ, Holtmann G, Agréus L, Jones M. Gastrointestinal symptoms and subjects cluster into distinct upper and lower groupings in the community: a four nations study. Am J Gastroenterol 2000;95:1439-1447.

6. Drossman DA. Functional gastrointestinal disorders: history, pathophysiology, clinical features and rome IV. Gastroenterology 2016;150:12621279 .

7. Malagelada JR, Accarino A, Azpiroz F. Bloating and abdominal distension: old misconceptions and current knowledge. Am J Gastroenterol 2017;112:1221-1231.

8. Accarino A, Perez F, Azpiroz F, Quiroga S, Malagelada JR. Abdominal distention results from caudo-ventral redistribution of contents. Gastroenterology 2009;136:1544-1551.

9. Guarner F, Malagelada JR. Gut flora in health and disease. Lancet 2003;361:512-519.

10. Mego M, Bendezú A, Accarino A, Malagelada JR, Azpiroz F. Intestinal gas homeostasis: disposal pathways. Neurogastroenterol Motil 2015;27:363-369.

11. Pimentel M, Lembo A, Chey WD, et al. Rifaximin therapy for patients with irritable bowel syndrome without constipation. N Engl J Med 2011;364:22-32.

12. Di Stefano M, Miceli E, Armellini E, Missanelli A, Corazza GR. Probiotics and functional abdominal bloating. J Clin Gastroenterol 2004;38(6 suppl):S102-S103.

13. Lewis SJ, Heaton KW. Stool form scale as a useful guide to intestinal transit time. Scand J Gastroenterol 1997;32:920-924.

14. Ghoshal UC, Ghoshal U, Das K, Misra A. Utility of hydrogen breath tests in diagnosis of small intestinal bacterial overgrowth in malabsorption syndrome and its relationship with oro-cecal transit time. Indian J Gastroenterol 2006;25:6-10.

15. Walters B, Vanner SJ. Detection of bacterial overgrowth in IBS using the lactulose $\mathrm{H} 2$ breath test: comparison with 14C-D-xylose and healthy controls. Am J Gastroenterol 2005;100:1566-1570.

16. Caporaso JG, Kuczynski J, Stombaugh J, et al. QIIME allows analysis of high-throughput community sequencing data. Nat Methods 2010;7:335336.

17. Becker RA, Chambers JM, Wilks AR. The new s language: a programming environment for data analysis and graphics. Pacific Grove: Chapman \& Hall 1988

18. Mardia KV, Kent JT, Bibby JM. Multivariate analysis. London-New York-Toronto-Sydney-San Francisco: Academic Press1979.

19. Martin N, Maes H. Multivariate analysis. London: Academic Press 1979.

20. Ondov BD, Bergman NH, Phillippy AM. Interactive metagenomic visualization in a web browser. BMC Bioinformatics 2011;12:385.

21. Bauer DF. Constructing confidence sets using rank statistics. J Am Stat Assoc 1972;67:687-690.

22. Hollander M, Wolfe DA, Chicken E. Nonparametric statistical methods. Hoboken:John Wiley \& Sons 2013.

23. Segata N, Izard J, Waldron L, et al. Metagenomic biomarker discovery 
and explanation. Genome Biol 2011;12:R60.

24. Lacy BE, Mearin F, Chang L, et al. Bowel disorders. Gastroenterology 2016;150:1393-1407, e5.

25. King TS, Elia M, Hunter JO. Abnormal colonic fermentation in irritable bowel syndrome. Lancet 1998;352:1187-1189.

26. Ringel-Kulka T, Benson AK, Carroll IM, Kim JH, Legge RM, Ringel Y. Molecular characterization of the intestinal microbiota in patients with and without abdominal bloating. Am J Physiol Gastrointest Liver Physiol 2016;310:G417-G426.

27. Jousimies-Somer HR. Update on the taxonomy and the clinical and laboratory characteristics of pigmented anaerobic gram-negative rods. Clin Infect Dis 1995;20(suppl 2):S187-S191.

28. Larsen JM. The immune response to Prevotella bacteria in chronic inflammatory disease. Immunology 2017;151:363-374.

29. Sokol H, Pigneur B, Watterlot L, et al. Faecalibacterium prausnitzii is an anti-inflammatory commensal bacterium identified by gut microbiota analysis of Crohn disease patients. Proc Natl Acad Sci USA 2008;105:16731-16736.

30. Sokol H, Seksik P, Furet JP, et al. Low counts of Faecalibacterium praus- nitzii in colitis microbiota. Inflamm Bowel Dis 2009;15:1183-1189.

31. Quévrain E, Maubert MA, Michon C, et al. Identification of an antiinflammatory protein from Faecalibacterium prausnitzii, a commensal bacterium deficient in Crohn's disease. Gut 2016;65:415-425.

32. Cano PG, Santacruz A, Moya Á, Sanz Y. Bacteroides uniformis CECT 7771 ameliorates metabolic and immunological dysfunction in mice with high-fat-diet induced obesity. PLoS ONE 2012;7:e41079.

33. Scharek L, Hartmann L, Heinevetter L, Blaut M. Bifidobacterium adolescentis modulates the specific immune response to another human gut bacterium, Bacteroiaes thetaiotaomicron, in gnotobiotic rats. Immunobiology 2000;202:429-441.

34. Odamaki T, Kato K, Sugahara H, et al. Age-related changes in gut microbiota composition from newborn to centenarian: a cross-sectional study. BMC Microbiol 2016;16:1-12.

35. Graf D, Di Cagno R, Fåk F, et al. Contribution of diet to the composition of the human gut microbiota. Microb Ecol Health Dis 2015;26:26164.

36. Azpiroz F, Malagelada JR. Abdominal bloating. Gastroenterology 2005;129:1060-1078. 